\title{
Características sensoriais e nutricionais de suplementos alimentares
}

\author{
Sensory and nutritional characteristics of dietary supplements
}

\author{
Mariah Benine Ramos Silva ${ }^{1}$; Hevelyse Munise Celestino dos Santos²; Ana Flávia \\ Oliveira $^{3}$
}

\begin{abstract}
Resumo
Suplementos alimentares são indicados quando a ingestão alimentar não supre as necessidades do organismo, situação que ocorre com maior frequência em pacientes com doenças infecciosas e crônicas, cujo gasto energético do organismo aumenta, no entanto o apetite diminui. Este estudo analisou as características sensoriais e nutricionais de sete diferentes marcas de suplementos alimentares completos não balanceados na versão em pó, em seus diversos sabores disponíveis, quatro para adultos e três para crianças, que possuem comercialização em âmbito nacional. A análise sensorial foi realizada por meio de testes afetivos. A análise das informações nutricionais foi realizada confrontando os dados presentes na rotulagem, comparando os produtos entre si. Observou-se grande diferença entre os teores de macro e micronutrientes nos suplementos alimentares estudados, podendo afirmar que os mesmos não são similares entre si. Os suplementos alimentares indicados para a faixa pediátrica apresentaram bom índice de aceitabilidade e são mais doces e saborosos quando comparados aos suplementos alimentares indicados para uso em adultos. Dois produtos não foram considerados bem aceitos em todos os sabores disponíveis. Os sabores chocolate e morango foram os mais bem aceitos e o sabor banana mal aceito entre os provadores.
\end{abstract}

Palavras-chave: Suplemento alimentar. Análise sensorial. Análise nutricional de alimentos.

\begin{abstract}
Dietary supplements are indicated when food intake is less than the nutritional requirement. This situation occurs most frequently in patients with infectious and chronic diseases, which increases the body's energy expenditure, but appetite decreases. This study examined the sensory and nutritional characteristics of seven different brands of complete unbalanced dietary supplements the powdered version, available in several flavors, four adults and three children, with marketing nationwide. Sensory analysis was performed by means of affective tests. We evaluated the data of nutritional content present on product labels for analysis of nutritional information. We observed a large difference between the levels of macro and micronutrients in food supplements and can say that they are not similar to each other. Food supplements indicated for pediatric patients had good index of acceptability and are sweeter and flavorful compared to food supplements indicated for use in adults. Two products were not considered well accepted in all flavors available. The chocolate and strawberry flavors were the best accepted and the banana flavor poorly accepted among tasters.
\end{abstract}

Key words: Dietary supplement. Sensory analysis. Nutritional analysis of foods.

\footnotetext{
${ }^{1}$ Mestranda em Ciência de Alimentos pela Universidade Estadual de Londrina. E-mail: mariah_benine@hotmail.com

${ }^{2}$ Doutoranda em Ciência de Alimentos pela Universidade Estadual de Maringá. E-mail: lyse_munise@yahoo.com.br

${ }^{3}$ Doutora em Nutrição pela Universidade Federal de São Paulo, Brasil. Professora da Universidade Tecnológica Federal do Paraná, Brasil. E-mail: anaflavia_nutri@hotmail.com
} 


\section{Introdução}

A alimentação pela via oral é a mais fisiológica e deve ser a preferida para a nutrição humana. $\mathrm{Na}$ presença de uma ou mais doenças instaladas, este cenário modifica-se significativamente, em especial naquelas doenças cujos sinais e sintomas alteram o comportamento alimentar, como as infecciosas agudas e crônicas, em que as necessidades nutricionais e energéticas aumentam, porém, o apetite diminui (BAXTER; WAITZBERG, 2006). Quando o paciente possui o trato gastrintestinal íntegro e consegue se alimentar, porém, não atinge suas necessidades orgânicas e perde peso, tem-se a indicação da introdução de suplementos alimentares, para auxiliar na recuperação do estado nutricional.

Os suplementos alimentares podem ser modulares, com somente um nutriente (carboidrato, proteína, etc.), incompletos (com alguns nutrientes) ou completos (com todos os nutrientes essenciais ao organismo humano). Podem ter seus nutrientes balanceados ou não, bem como modificados conforme as necessidades dietoterápicas da doença em tratamento. Podem, ainda, ser utilizado como única fonte de alimentação (denominados como fórmulas enterais) ou como complementação.

A indústria farmacêutica disponibiliza vários suplementos alimentares para ingestão oral, com objetivo de complementar a ingestão alimentar ou substituí-la totalmente. Existem modelos prontos para consumo, vendidos em tetra-pack e latas, ou em pó, necessitando ser diluído antes de consumido (VASCONCELOS, 2002), no entanto, ainda é comum o relato de pacientes que interrompem o seu uso por não suportarem mais a monotonia dos seus sabores (BAXTER; WAITZBERG, 2006).

Apesar de alguns estudos apontarem o crescimento do uso de suplementos alimentares ou fórmulas completas em outros países, não há dados sobre essa situação no Brasil. Temos um valor aproximado de consumo de suplementos orais em hospitais brasileiros, que, conforme o Inquérito Brasileiro de Avaliação Nutricional
Hospitalar (IBRANUTRI), publicado em 2001, do total de 4000 pacientes, o consumo de suplementos nutricionais orais foi observado em apenas $4 \%$ deles (WAITZBERG; CAIAFFA; CORREIA, 2001).

Os suplementos alimentares para uso via oral têm enorme potencial de utilização para prevenção ou tratamento de pacientes com algum grau ou em risco de desnutrição, sejam hospitalizados ou em cuidado domiciliar. Dessa forma, este trabalho teve por objetivo avaliar as características sensoriais e nutricionais dos suplementos alimentares completos não balanceados disponíveis no mercado nacional, uma vez que esses dados são escassos na literatura científica, no entanto, importantes para a prática clínica.

\section{Material e Métodos}

Foram avaliados sete suplementos alimentares completos não balanceados, na versão pó, comercializados nacionalmente, sendo quatro indicados para uso de adultos e três para crianças; de acordo com os sabores disponíveis no mercado: chocolate, morango, baunilha e banana; adquiridos nos supermercados e farmácias da cidade de Londrina, PR.

Para avaliação das características nutricionais de cada produto, retiraram-se os dados dos nutrientes informados na rotulagem dos produtos, comparando-os entre si, de acordo com a medida caseira para uma porção, todos equivalente a duas colheres de sopa. O peso do suplemento para esta porção variou de 30 a $40 \mathrm{~g}$ de cada produto. Optouse por não padronizar o peso da porção, pois o consumidor, em geral, segue a orientação do rótulo do produto. Os nutrientes avaliados foram: ferro, zinco, vitamina A, vitamina $\mathrm{C}$, carboidrato, fibra, proteína e lipídeos.

A análise sensorial foi realizada por meio dos seguintes testes afetivos: aceitação, índice de aceitabilidade e escala de intensidade. No teste de aceitação as amostras foram avaliadas quanto à 
aceitação global (análise do produto como um todo), utilizando-se de uma escala hedônica estruturada de 7 pontos ( 1 = desgostei muito, $7=$ gostei muito). Com base na nota média de aceitação global, calculou-se o índice de aceitabilidade (IA) do produto (média da aceitação global x 100 / 7), sendo considerado o mínimo de $70 \%$ de IA como limite para considerar que o produto fosse bem aceito pelos consumidores (DUTCOSKY, 2007).

Os testes usando escalas indicam o tipo ou a intensidade de uma resposta sensorial e são utilizadas nas avaliações de atributos específicos dos produtos por meio de uma escala pré-definida (INSTITUTO ADOLFO LUTZ, 2008). Os provadores avaliaram a intensidade dos atributos: cor, aroma, sabor, doçura e gosto residual metálico por meio de uma escala de intervalo estruturada verbal numérica de 5 pontos (1 $=$ muito fraca, $5=$ muito forte).

Os testes sensoriais foram conduzidos no laboratório de Análise Sensorial da Universidade Tecnológica Federal do Paraná, campus Londrina. Os suplementos foram preparados, quanto às quantidades de pó e de leite integral, de acordo com o modo de preparo informado em seu rótulo. As amostras, codificadas com número de três dígitos, foram dispostas em blocos incompletos casualizados, em copos descartáveis, contendo $30 \mathrm{ml}$ de cada produto por sabor. Em cada teste sensorial (bloco), avaliou-se uma marca com todos os seus sabores disponíveis. A equipe foi formada por 50 provadores voluntários, entre alunos e os professores da instituição, de ambos os sexos, com idade entre 18 a 46 anos.

O presente trabalho foi aprovado pelo Comitê de Bioética e Ética em Pesquisa da Irmandade Santa Casa de Londrina, protocolo 028/09, para a realização dos testes sensoriais. Os provadores foram orientados quanto aos testes sensoriais e assinaram o termo de consentimento livre esclarecido antes de provarem as amostras.

Para apresentação dos resultados, os suplementos foram codificados em letras, a fim de preservar as marcas. Os dados da avaliação das características nutricionais foram apresentados em tabelas descritivas. Os resultados do teste de aceitação e escala de intensidade foram analisados por Análise de Variância (ANOVA), seguido do teste de média pos hoc de Tukey, com o auxílio do programa BioEstat $^{\circledR}$. Adotou-se o nível de significância de 5\% $(\mathrm{p}<0,05)$.

\section{Resultados e Discussão}

A tabela 1 apresenta os teores de ferro, zinco, vitamina A, vitamina $\mathrm{C}$, carboidrato, fibra, proteína e lipídeos, de acordo com a porção individual informada nos rótulos dos suplementos avaliados. Avaliando as características nutricionais dos suplementos alimentares, observa-se que a marca A foi a que apresentou maiores teores de nutrientes por porção individual, além de ofertar maior valor energético comparado às demais.

Tabela 1 - Teor de ferro, zinco, vitamina A, vitamina C, carboidrato, fibra, proteína e lipídeos, de acordo com a porção individual informada nos rótulos dos suplementos avaliados, Londrina, 2009-2010.

\begin{tabular}{llllllll}
\hline Nutriente & Marca A & Marca A Jr & Marca B & Marca B Jr & Marca C & Marca C Jr & Marca D \\
\hline Porção $(\mathrm{g})$ & 40 & 30 & 40 & 40 & 30 & 30 & 31,5 \\
Ferro (mg) & 7,2 & 5,9 & 2,6 & 4 & 14 & 6 & 6,3 \\
Zinco (mg) & 4,8 & 4,3 & 2,6 & 2,6 & ND & 0,05 & 3,2 \\
Vitamina A (mcg) & 342 & 133 & 240 & 45 & 179 & 150 & 228 \\
Vitamina C (mg) & 40 & 30 & 18 & 22 & 21 & 14 & 20 \\
Carboidrato (g) & 26 & 27 & 36 & 36 & 22 & 26 & 18 \\
Fibra (g) & 0 & 0 & 0 & 0 & 0 & 0 & 1,7 \\
Proteína (g) & 9,7 & 1,3 & 2,4 & 2,2 & 4,8 & 1,9 & 7,6 \\
Lipideos (g) & 1,5 & 0 & 0 & 0 & 0,5 & 0 & 1 \\
Valor Energético & 154 & 114 & 150 & 152 & 111 & 118 & 112
\end{tabular}

(Kcal)

'Porção para consumo individual, por vez, do produto. Todos correspondiam a duas colheres de sopa como medida caseira.

2ND: Não disponível no rótulo.

Fonte: Autoras.

$\mathrm{O}$ ferro esteve presente em maior quantidade na marca C, seguido das marcas A e D. As funções mais importantes desse nutriente estão relacionadas às ações das proteínas que contém ferro no organismo, como as que contêm heme 
(hemoglobina, mioglobina e citocromos) e as de transporte e armazenamento como a transferrina, ferritina e hemossiderina (HENRIQUES; COZZOLINO, 2007). Sabe-se que sua deficiência pode causar anemia ferropriva, como problema de saúde pública, (SIQUEIRA; ALMEIDA; ARRUDA, 2006), no entanto, há fortes evidências que a carência de ferro está associada a uma diminuição da atividade bactericida dos leucócitos polimorfonucleares e a um déficit na resposta imune celular especialmente contra $S$ aureus e $C$ albicans. Assim, seu balanço normal deve ser idealizado, evitando-se elevações acentuadas de ferro sérico e da saturação de transferrina de forma que o organismo obtenha uma quantidade suficiente de ferro para permitir uma resposta imune adequada e, ao mesmo tempo, não excessiva para aumentar o ferro disponível às bactérias para sua multiplicação (BRICKS, 1994).

$\mathrm{O}$ zinco apresentou maior quantidade nas marcas A e A Jr e esteve ausente apenas na marca C. As funções do zinco podem ser divididas em três categorias: estrutural (determinante da forma e da disposição espacial de enzimas e proteínas), enzimática (aproximadamente 300 enzimas necessitam de zinco para sua ação catalítica) e reguladora (elemento necessário para síntese proteica, replicação de ácidos nucleicos, divisão celular, etc.). São alguns dos sinais da deficiência em zinco: alterações no apetite e perda do paladar. Como este nutriente desempenha função primordial na transcrição de polinucleotídios e, consequentemente, na regulação da expressão gênica, sua deficiência pode trazer danos no crescimento e reparo celular (YUYAMA et al., 2009).

A vitamina A esteve presente em todas as marcas sendo que a marca $A$, seguidas da marca $B$ e D possuem as maiores quantidades. Segundo Silva e Mura (2007) a vitamina A, conhecida como retinol, desempenha funções básicas no organismo, atuando no crescimento, na visão, na integridade estrutural e funcional dos epitélios, no processo da reprodução e sobre os dentes.

A vitamina $\mathrm{C}$ também esteve presente em todas as marcas, sendo que as marcas A e A Jr possuem as maiores quantidades. Esta vitamina é conhecida pela sua ação antioxidante, mas também atua como co-fator ou co-substrato para enzimas, além de fazer parte da primeira linha de defesa do organismo e ser capaz de aumentar a absorção do ferro (SILVA; COZZOLINO, 2009).

As quantidades de carboidratos são maiores nos suplementos B e B Jr. Os carboidratos são a principal fonte de energia para a maioria das células do organismo. Além de sua função energética, os carboidratos têm também uma função poupadora de proteínas, pois uma quantidade suficiente de carboidratos impede que as proteínas corpóreas sejam utilizadas como fonte de energia mantendo-as em sua função estrutural (WAITZBERG; GALIZIA, 2006).

Apenas a marca D apresentou fibra em seu conteúdo. É importante ressaltar que os suplementos minerais são, em geral, obstipantes e, diante disso, a ingestão de fibras são importantes devido ao seu efeito laxativo. As fibras apresentam ação prebiótica, ou seja, não são digeridas no intestino delgado e ao atingir o intestino grosso são metabolizados por bactérias benéficas que alteram a microflora intestinal induzindo efeitos fisiológicos importantes para a saúde (COLLI; SARDINHA; FILISETTI, 2002). As fibras presentes nesse suplemento em questão foram a inulina e o frutooligossacarídeo que, ao serem fermentados no intestino, promovem o crescimento de bactérias dos gêneros Bifidobacterium e Lactobacillus capazes de competir favoravelmente com bactérias patogênicas como Enterococcus e Clostridium, produção de ácidos graxos de cadeia curta e aumento da massa fecal (COUTINHO; MENDES; ROGERO, 2007). 
As marcas A e D foram as que apresentam maior quantidade de proteínas. As proteínas cumprem funções estruturais, reguladoras, de defesa e de transporte nos fluidos biológicos. Um dos principais problemas ocasionado pela ingestão inadequada de proteínas, associadas à baixa ingestão energética, é a desnutrição energético protéica (DEP) que provêm da falta, em várias proporções de proteínas e energia (TIRAPEGUI; CASTRO; ROSSI, 2009).

Apenas três marcas apresentaram lipídeos em sua composição, são elas A, C e D, sendo que a A apresentou maior quantidade deste nutriente. Os lipídeos desempenham várias funções no organismo, desde a composição de membranas celulares e proteção de órgão vital até a participação ativa na resposta imunológica por comportar-se como precursor de prostaglandinas (BAXTER; BORGHI, 2010).

Como estes produtos são complementares à alimentação, não é possível afirmar que um possa ser melhor que o outro, pois este dependerá dos demais alimentos que fazem parte da dieta do paciente. No entanto, pode-se afirmar que os mesmos não são similares entre si, uma vez que a diferença entre os teores de macro e micronutrientes é grande. Este dado é importante, uma vez que o usuário de um produto, não deveria trocar por outro, sem a prescrição do seu nutricionista ou médico.

Observa-se na tabela 2 que os sabores chocolate e morango foram os que apresentaram melhores médias de aceitação e obtiveram os maiores índices de aceitabilidade. Segundo Dutcosky (2007), o produto para ser bem aceito deve ter IA mínimo de 70\%. A Marca A e D apresentaram os piores IA, portanto, são produtos com maior possibilidade de rejeição pelos seus consumidores. Interessante observar que todos os suplementos alimentares elaborados para a faixa etária pediátrica apresentaram bons índices de IA.
Tabela 2 - Média e desvio-padrão da aceitação global e seu respectivo índice de aceitabilidade (IA) dos suplementos alimentares, conforme o sabor disponível de cada marca avaliada, Londrina, 20092010.

\begin{tabular}{|c|c|c|c|c|}
\hline Suplementos & Chocolate & Morango & Baunilha & Banana \\
\hline Marca A & $3,8^{\mathrm{b}} \pm 1,7$ & $4,7^{\mathrm{a}} \pm 1,7$ & $3,0^{\mathrm{c}} \pm 2,0$ & $3,1^{\mathrm{c}} \pm 1,7$ \\
\hline IA \% & 54 & 67 & 43 & 44 \\
\hline Marca A Jr & $5,3^{a} \pm 1,5$ & $5,6^{a} \pm 1,2$ & $4,6^{b} \pm 1,3$ & \\
\hline $\mathrm{IA} \%$ & 76 & 80 & 66 & --------- \\
\hline Marca B & $5,7^{a} \pm 1,3$ & $5,1^{\mathrm{b}} \pm 1,5$ & $5,9^{\mathrm{a}} \pm 1,2$ & \\
\hline IA\% $\%$ & 81 & 73 & 84 & \\
\hline Marca B Jr & $5,1^{c} \pm 1,7$ & $5,3^{\mathrm{b}} \pm 1,0$ & $5,7^{\mathrm{a}} \pm 1,1$ & \\
\hline $\mathrm{IA} \%$ & 73 & 76 & 81 & \\
\hline Marca C & $4,8^{a} \pm 1,6$ & $4,6^{a} \pm 2,0$ & $2,4^{\mathrm{b}} \pm 1,8$ & ------ \\
\hline $\mathrm{IA} \%$ & 69 & 66 & 34 & ----------- \\
\hline Marca C Jr & $5,5^{\mathrm{a}} \pm 1,3$ & $4,9^{\mathrm{b}} \pm 1,4$ & $5,7^{\mathrm{a}} \pm 1,2$ & ---------- \\
\hline $\mathrm{IA} \%$ & 79 & 70 & 81 & --------- \\
\hline Marca D & $5,1^{a} \pm 1,5$ & $4,6^{b} \pm 1,8$ & $4,1^{\mathrm{c}} \pm 1,9$ & $3,7^{d} \pm 1,7$ \\
\hline $\mathrm{IA} \%$ & 73 & 66 & 59 & 53 \\
\hline
\end{tabular}

Escala: 1 = desgostei muito; 7 = gostei muito

Médias seguidas pelas mesmas letras nas linhas não diferem entre si pelo Teste de Tukey $(\mathrm{p}<0,05)$.

Fonte: Autoras.

Encontrou-se grande variação da aceitação global dos produtos, de acordo com o sabor disponível. $\mathrm{Na}$ marca A e A Jr, o mais bem aceito foi o sabor morango seguido do chocolate; na marca B e B Jr o sabor baunilha prevaleceu, seguido do morango; na marca $\mathrm{C}$ o sabor chocolate foi mais bem aceito, seguido do morango, no entanto, na C Jr o sabor baunilha seguido do chocolate foram os sabores mais bem aceitos; por fim, na marca D o sabor chocolate obteve melhor aceitação. O sabor banana foi o menos aceito e presente em um único produto disponível no mercado. O sabor baunilha, embora nem sempre bem aceito, pode ser interessante, pois como seu sabor é menos marcante, pode ser utilizado no preparo de outros alimentos, como vitaminas de frutas, shakes, etc.

O suplemento alimentar da marca A foi o menos aceito na avaliação do IA de todos os sabores, no entanto foi o que apresentou maiores teores de nutrientes. Não basta um suplemento conter bons 
teores de nutrientes se não tiver uma boa aceitação. Cabe lembrar que a análise sensorial é realizada com apenas $30 \mathrm{ml}$ de produto, no entanto, a porção mínima individual é de $200 \mathrm{ml}$ de produto preparado ao dia. Um produto mal aceito não será utilizado da maneira prescrita pelo profissional, não atingindo suas funções de complementar a alimentação diária.

A tabela 3 apresenta as notas dadas aos suplementos, de acordo com os atributos avaliados na escala de intensidade: cor, aroma, sabor, doçura e gosto residual metálico; conforme o sabor disponível de cada marca avaliada. As marcas A Jr, $\mathrm{B}$, B Jr e C Jr foram as que apresentaram melhores médias para os atributos cor, aroma, sabor e doçura.

Tabela 3 - Média e desvio-padrão das notas dadas aos suplementos, de acordo com os atributos avaliados na escala de intensidade, conforme o sabor disponível de cada marca avaliada, Londrina, 2009-2010.

\begin{tabular}{|c|c|c|c|c|c|c|c|}
\hline Atributos & Marca A & Marca $\mathrm{A} \mathrm{J}_{\mathrm{I}}$ & Marca B & Marca BJIr & Marca C & Marca C.Jr & Marca D \\
\hline \multicolumn{8}{|l|}{ Cor } \\
\hline Chocolate & $2,5 \pm 1^{\mathrm{a}}$ & $3,3 \pm 1,0^{b}$ & $4,1 \pm 0,8^{c}$ & $3,6 \pm 1,0^{\mathrm{c}}$ & $2,9 \pm 0,8^{\mathrm{a}}$ & $4,0 \pm 0,6^{\mathrm{c}}$ & $3,3 \pm 0,9^{\mathrm{b}}$ \\
\hline Morango & $2,9 \pm 0,8^{\mathrm{a}}$ & $4,1 \pm 0,7^{b}$ & $4,2 \pm 0,7^{\mathrm{b}}$ & $4,0 \pm 0,8^{b}$ & $3,2 \pm 0,8^{\mathrm{a}}$ & $4,2 \pm 0,8^{b}$ & $3,4 \pm 0,7^{\mathrm{a}}$ \\
\hline Baunilha & $2,7 \pm 0,8^{a}$ & $3,2 \pm 0,8^{\mathrm{a}}$ & $3,4 \pm 0,8^{b}$ & $3,2 \pm 0,8^{\mathrm{a}}$ & $3,2 \pm 0,8^{\mathrm{a}}$ & $3, \mathbf{I} \pm 0,8^{a}$ & $3,2 \pm 0,9^{\mathrm{a}}$ \\
\hline Banana & $3,4 \pm 0,8^{\mathrm{a}}$ & -..-- & - & -- & - & -...- & $3,0 \pm 0,8^{b}$ \\
\hline \multicolumn{8}{|l|}{ Aroma } \\
\hline Chocolate & $3,1 \pm 0,8^{a}$ & $3,3 \pm 1,0^{\mathrm{a}}$ & $4,0 \pm 0,9^{b}$ & $3,5 \pm 1,0^{\mathrm{a}}$ & $2,8 \pm 0,9^{c}$ & $3,4 \pm 1,0^{a}$ & $3, \mathbf{1} \pm \mathbf{1}, 0^{\mathrm{a}}$ \\
\hline Morango & $3,2 \pm 0,9^{\mathrm{a}}$ & $3,7 \pm 0,8^{\mathrm{a}}$ & $3,7 \pm 0,9^{\mathrm{a}}$ & $3,7 \pm 0,8^{\mathrm{a}}$ & $3,2 \pm 0,9^{\mathrm{a}}$ & $3,3 \pm 0,9^{a}$ & $3,2 \pm \mathbf{1}, 0^{\mathrm{a}}$ \\
\hline Baunilha & $2,4 \pm 1,0^{\mathrm{a}}$ & $2,7 \pm 0,8^{\mathrm{a}}$ & $3,5 \pm 0,9^{\mathrm{b}}$ & $3,2 \pm 0,8^{\mathrm{c}}$ & $2,9 \pm 0,9^{\mathrm{a}}$ & $3,3 \pm 0,8^{c}$ & $2,5 \pm 1,2^{\mathrm{a}}$ \\
\hline Banana & $2,8 \pm 1,1^{\mathrm{a}}$ & --.-- & --.-- & -..- & -..-- & -...- & $2,8 \pm 1,0^{\mathrm{a}}$ \\
\hline \multicolumn{8}{|l|}{ Sabor } \\
\hline Chocolate & $2,7 \pm 1,1^{a}$ & $3,7 \pm \mathbf{1}, 0^{\mathrm{b}}$ & $4,0 \pm 0,8^{c}$ & $3,5 \pm 0,9^{b}$ & $2,7 \pm 1,0^{\mathrm{a}}$ & $3,5 \pm 1,0^{b}$ & $2,7 \pm 0,9^{\mathrm{a}}$ \\
\hline Morango & $3,1 \pm 1,0^{\mathrm{a}}$ & $3,8 \pm 0,8^{\mathrm{b}}$ & $3,8 \pm 0,9^{b}$ & $3,5 \pm 1,0^{c}$ & $2,9 \pm 1,1^{a}$ & $3,2 \pm 0,9^{\mathrm{a}}$ & $2,9 \pm 1,0^{\mathrm{a}}$ \\
\hline Baunilha & $2,3 \pm 1,2^{\mathrm{a}}$ & $2,9 \pm 1,0^{\mathrm{b}}$ & $3,6 \pm 0,8^{c}$ & $3,7 \pm 0,9^{c}$ & $1,6 \pm 0,8^{\mathrm{d}}$ & $3,5 \pm 1,0^{c}$ & $2,7 \pm \mathbf{1}, 1^{\mathrm{b}}$ \\
\hline Banana & $2,3 \pm 1,2^{\mathrm{a}}$ & -...-... & -...- & -...... & -.--..--.. & -..--..- & $2,8 \pm 1,1^{\mathrm{b}}$ \\
\hline \multicolumn{8}{|l|}{ Doçura } \\
\hline$\overline{\text { Chocolate }}$ & $2,5 \pm 0,9^{\mathrm{a}}$ & $3,6 \pm 0,7^{b}$ & $3,5 \pm 0,7^{\mathrm{b}}$ & $3,2 \pm 0,8^{\mathrm{c}}$ & $2,6 \pm 0,8^{a}$ & $3,1 \pm 0,9^{c}$ & $2,9 \pm 0,9^{c}$ \\
\hline Morango & $3,2 \pm \mathbf{1}, 0^{\mathrm{a}}$ & $4,0 \pm 0,8^{b}$ & $3,7 \pm 0,9^{b}$ & $3,4 \pm 1,0^{\mathrm{a}}$ & $2,6 \pm 1,0^{\mathrm{c}}$ & $3,4 \pm 0,8^{\mathrm{a}}$ & $2,4 \pm 1,0^{\mathrm{c}}$ \\
\hline Baunilha & $2,0 \pm 0,8^{\mathrm{a}}$ & $3,6 \pm 0,8^{\mathrm{b}}$ & $3,8 \pm 0,8^{\mathrm{b}}$ & $3,8 \pm 0,9^{\mathrm{b}}$ & $1,5 \pm 0,6^{\mathrm{c}}$ & $3,5 \pm 1,0^{\mathrm{a}}$ & $2,6 \pm 0,9^{\mathrm{d}}$ \\
\hline Banana & $2,1 \pm 0,8^{\mathrm{a}}$ & --.-- & - & --.-- & ---- & - & $2,4 \pm 1,0^{\mathrm{b}}$ \\
\hline \multicolumn{8}{|c|}{$\begin{array}{l}\text { Gosto residual } \\
\text { metálico }\end{array}$} \\
\hline $\begin{array}{l}\text { Chocolate } \\
\text { Morango }\end{array}$ & $\begin{array}{l}2,7 \pm 1,2^{\mathrm{a}} \\
3,2 \pm 1,0^{\mathrm{a}}\end{array}$ & $\begin{array}{l}2,5 \pm 1,4^{b} \\
4,0 \pm 0,8^{b}\end{array}$ & $\begin{array}{l}3,0 \pm 1,3^{\mathrm{c}} \\
3,7 \pm 0,9^{\mathrm{b}}\end{array}$ & $\begin{array}{l}2,9 \pm 1,3^{\mathrm{c}} \\
3,4 \pm 1,0^{\mathrm{a}}\end{array}$ & $\begin{array}{l}2,8 \pm 1,2^{\mathrm{a}} \\
2,6 \pm 1,0^{\mathrm{c}}\end{array}$ & $\begin{array}{l}2,7 \pm 1,3^{\mathrm{a}} \\
3,4 \pm 0,8^{\mathrm{a}}\end{array}$ & $\begin{array}{l}2,4 \pm 1,1^{b} \\
2,4 \pm 1,0^{c}\end{array}$ \\
\hline Baunilha & $2,0 \pm 0,8^{\mathrm{a}}$ & $3,6 \pm 0,8^{b}$ & $3,8 \pm 0,8^{\mathrm{b}}$ & $3,8 \pm 0,9^{b}$ & $1,5 \pm 0,6^{\mathrm{c}}$ & $3,5 \pm 1,0^{\mathrm{a}}$ & $2,6 \pm 0,9^{\mathrm{d}}$ \\
\hline Banana & $2,1 \pm 0,8^{a}$ & 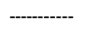 & -.---.- & --.-- & -.------ & -..---- & $2,4 \pm 1,0^{\mathrm{b}}$ \\
\hline \multicolumn{8}{|c|}{$\begin{array}{l}\text { Gosto residual } \\
\text { metálico }\end{array}$} \\
\hline Chocolate & $2.7 \pm 1.2^{a}$ & $2,5 \pm 1.4^{b}$ & $3,0 \pm 1,3^{\circ}$ & $2,9 \pm 1,3^{\circ}$ & $2,8 \pm 1.2^{\mathrm{a}}$ & $2,7 \pm 1,3^{a}$ & $2,4 \pm 1,1^{\mathrm{b}}$ \\
\hline Morango & $2,6 \pm 1,2^{a}$ & $2,4 \pm 1,2^{\mathrm{a}}$ & $3,0 \pm 1,4^{b}$ & $2,6 \pm 1,3^{a}$ & $2,8 \pm 1,2^{b}$ & $3,1 \pm 1,3^{\mathrm{a}}$ & $2,3 \pm \mathbf{1}, 0^{\mathrm{c}}$ \\
\hline Baunilha & $2,7 \pm 1,3^{\mathrm{a}}$ & $2,4 \pm 1,2^{b}$ & $2,5 \pm 1,2^{b}$ & $2,4 \pm 1,1^{b}$ & $2,8 \pm 1,2^{\mathrm{a}}$ & $2,6 \pm 1,2^{a}$ & $2,5 \pm 0,9^{b}$ \\
\hline Banana & $2.8 \pm 1.3^{a}$ & -- & - & - & -- & -...- & $2,7 \pm 1,2^{\mathrm{a}}$ \\
\hline
\end{tabular}

Escala: $1=$ muito fraco; $5=$ muito forte.

Médias seguidas pelas mesmas letras nas linhas não diferem entre si pelo Teste de Tukey $(\mathrm{p}<0,05)$.

Fonte: Autoras.
Nos atributos cor, aroma e sabor as marcas avaliadas deixaram a desejar, pois as médias variaram entre 2 (fraco) e 3 (regular) com algumas exceções que apresentaram média 4 (forte). $\mathrm{O}$ conjunto desses atributos deveria ser mais intenso, uma vez que pacientes em processos infecciosos apresentam anorexia transitória, o que reduz a vontade de comer. Este fato decorre da resposta inflamatória da doença, pois nesta situação o organismo libera citocinas pró-inflamatórias, como a interleucina 6 (IL-6), que age diretamente no sistema nervoso, fazendo com que haja perda da fome por meio da liberação do peptídeo anorexígeno e, com isso, a ingestão de alimentos fica prejudicada, acarretando a perda de peso. Assim, suplementos com cor, aroma e sabores mais intensos são mais interessantes para pacientes com doenças infecciosas agudas ou agudizadas (LEÃO; FORTE; CAMPOS, 1985; MENEZES; BERTOLA, 2001).

Segundo Dolce e Canavó (2006) os alimentos e preparações devem oferecer um bom estímulo visual e odorífero por meio da sua apresentação, portanto, a cor e o aroma são atributos importantes a serem considerados na aceitação do produto antes dele ser consumido, uma vez que a primeira impressão que temos ao olhar para o alimento diz respeito à sua aparência e ao seu aroma.

A marca A não apresentou diferença significativa em todos os atributos e sabores avaliados, sendo sensorialmente semelhantes entre si $(p>0,05)$. Nos atributos sabor e doçura as marcas A Jr, B, B Jr e C Jr obtiveram as maiores médias. É interessante que os suplementos tenham maior intensidade de sabor e doçura, pois indivíduos desnutridos têm menor palatabilidade, o que, consequentemente, reduz a ingestão alimentar, sendo estes os maiores candidatos ao uso de suplementos alimentares (BAXTER; WAITZBERG, 2006).

Ressalta-se que as três marcas para o público infantil são mais doces e têm sabores mais bem aceitos, mostrando que a indústria se preocupa mais com este público do que os adultos. Isto pode ser 
atribuído ao fato de que muitas mães, por crerem que seus filhos não comem bem, ofertam os suplementos alimentares aos seus filhos para que haja uma compensação alimentar. Assim, um produto mais saboroso tem maiores possibilidades de venda no comércio, uma vez que estes suplementos alimentares não necessitam de receita médica e são encontrados à venda para o público em mercados e farmácias.

O atributo gosto residual metálico diz respeito ao gosto que os minerais contidos nos produtos deixam na boca após sua ingestão. As médias foram baixas para este atributo, sendo este fator considerado positivo, ou seja, esta característica não foi marcante nos suplementos alimentares avaliados.

A terapia nutricional, incluindo o uso de suplementos alimentares, é parte importante do tratamento de pacientes com ingestão alimentar abaixo das necessidades nutricionais. Estudos realizados em ambiente hospitalar mostram que o uso de suplementos alimentares tem efeito benéfico nos pacientes, melhorando seu estado nutricional quando comparados a pacientes que não receberam essa suplementação (STRATTON; ELIA, 2000). No entanto, cabe ressaltar que no ambiente hospitalar existe a possibilidade de controle da ingestão de tais suplementos, mas em ambiente residencial esse controle é nulo, cabendo então, aos profissionais de saúde, prescreverem produtos que sejam, além de nutritivos, bem aceitos pelos pacientes, para que haja seu real consumo e produza respostas nutricionais efetivas.

\section{Conclusões}

Observou-se grande diferença entre os teores de macro e micronutrientes nos suplementos alimentares estudados, podendo afirmar que os mesmos não são similares entre si. $\mathrm{O}$ suplemento alimentar da marca A foi o que apresentou maior teor de nutrientes, porém seu índice de aceitabilidade foi o pior entre as marcas avaliadas. Por outro lado, as marcas B e B Jr obtiveram os melhores índices de aceitabilidade entre os suplementos analisados.

Os suplementos alimentares indicados para a faixa pediátrica obtiveram bom índice de aceitabilidade, são mais doces e saborosos. Dois produtos não foram considerados bem aceitos em todos os sabores disponíveis. Os sabores chocolate e morango foram os mais bem aceitos de maneira geral entre os produtos analisados, no entanto, o sabor banana foi mal aceito entre os provadores.

Ressalta-se a necessidade de melhor avaliação por parte dos profissionais de saúde, como médicos e nutricionistas, quanto aos suplementos alimentares prescritos aos seus pacientes, pois, embora com mesma indicação clínica: suplementação alimentar, seu conteúdo nutricional e suas características sensoriais são diferentes, o que pode levar ao insucesso terapêutico e piora do estado nutricional do paciente.

\section{Referências}

BAXTER, Y. C.; BORGHI, R. T. Nutrientes imunomoduladores e suas aplicações. In: SILVA, S. M. C. S.; MURA, J. D. P. Tratado de alimentação, nutrição e dietoterapia. São Paulo: Roca, 2010.

BAXTER, Y. C.; WAITZBERG, D. L. Indicações e usos de suplementos nutricionais orais. In: WAITZBERG, D. L. Nutrição oral, enteral e parenteral na prática clínica. 3 ed. São Paulo: Atheneu, 2006. p. 543-571.

BRICKS, L. F. Ferro e infecções: atualização. Pediatria, São Paulo, v. 16, n. 1, p.34-43, 1994.

COLLI, C.; SARDINHA, F.; FILISETTI, T. M. C. C. Alimentos funcionais. In: CUPPARI, L. Nutrição clínica no adulto. São Paulo: Manole, 2002.

COUTINHO, V. F.; MENDES, R. R.; ROGERO, M. M. Bioquímica e metabolismo dos carboidratos. In: SILVA, S. M. C. S.; MURA, J. D. P. Tratado de alimentação, nutrição e dietoterapia. São Paulo: Roca, 2007. p. 21-49. 
DOLCE, P.; CANAVÓ, P. R. L. Alimentação oral na criança enferma. In: WAITZBERG, D. L. Nutrição oral, enteral e parenteral na prática clínica. 3 ed. São Paulo: Atheneu, 2006. p. 433-447.

DUTCOSKY, S. D. Métodos subjetivos ou afetivos. In:_. Análise sensorial de alimentos. 2. ed. Curitiba: Champagnat, 2007. p. 141-173.

HENRIQUES, G. S.; COZZOLINO, S. M. F. In: COZZOLINO, S. M. F. Biodisponibilidade de nutrientes. 2. ed. Barueri: Manole, 2007. p. 508-432.

INSTITUTO ADOLFO LUTZ. Análise sensorial. In:_. Métodos físico-químicos para análise de alimentos. São Paulo, 2008. p. 278 - 320.

LEÃO, R. C.; FORTE, W. C. N.; CAMPOS, J. V. M. A imunidade inespecífica em crianças com má nutrição calórico-protéica moderada. Revista Brasileira de Alergia e Imunopatologia, São Paulo, v. 8, n. 1, p. 5-8, 1985.

MENEZES, H.; BERTOLA, E. A inter-relação entre nutrição e imunidade. Nutrição em Pauta, São Paulo, v. 49, p. 36-40, 2001.

SILVA, S. M. C. S.; MURA, J.P. Tratado de alimentação, nutrição e dietoterapia. São Paulo: Roca, 2007.

SILVA, V. L.; COZZOLINO, S. M. F. Vitamina C (ácido ascórbico). In: COZZOLINO, S. M. F. Biodisponibilidade de nutrientes. 3. ed. Barueri: Manole, 2009. p. 354-373.

SIQUEIRA, E. M. A.; ALMEIDA, S. G.; ARRUDA, S. Papel adverso do ferro no organismo. Comunicação em Ciências da Saúde, Brasília, v. 17, n. 3, p. 229-236, 2006.

STRATTON, R. J.; ELIA, M. How much undernutrition is there in hospital? British Journal of Nutrition, Cambridge, v. 84, p. 257-259, 2000.

TIRAPEGUI, J.; CASTRO, I. A.; ROSSI, L. Biodisponibilidade de proteínas. In: COZZOLINO, S. M. F. Biodisponibilidade de nutrientes. 3. ed. Barueri: Manole, 2009. p. 89-153.
VASCONCELOS, M. I. L. Nutrição enteral. In: CUPPARI, L. Nutrição clínica no adulto. São Paulo: Manole, 2002. p. 369-390.

WAITZBERG, D. L.; CAIAFFA, W. T.; CORREIA, M. I. Hospital malnutrition: the Brazilian national survey (IBRANUTRI): a study of 4000 patients. Nutrition, New York, v. 17, p. 573-580, 2001.

WAITZBERG, D. L.; GALIZIA, M. S. Carboidratos. In: WAITZBERG, D. L. Nutrição oral, enteral e parenteral na prática clínica. 3 ed. São Paulo: Atheneu, 2006. p. 15-33.

YUYAMA, L. K. O. YONEKURA, L.; AGUIAR, J. P. L.; RODRIGUES, M. L. C.; COZZOLINO, S. M. F. Zinco. In: COZZOLINO, S. M. F. Biodisponibilidade de nutrientes. 3. ed. Barueri: Manole, 2009. p. 616-643. 